\title{
UPDATE ON THE MANAGEMENT OF OSTEOARTHRITIS
}

Goh EML, Chow SK and Yeap SS

Department of Medicine, University of Malaya Medical Centre, 50603 Kuala Lumpur

\begin{abstract}
Osteoarthritis is the most common joint disorder in the human population. It is characterized by the destruction of articular cartilage and the overgrowth of marginal and subchondral bone. The commonest weight bearing joint affected is that of the knee, affecting over one third of people over the age of 65 years. This article reviews the recent principles of non surgical management of osteoarthritis. This is divided into non drug management such as primary prevention, physiotherapy, occupational therapy and patient education. Drug management will include the use of different types of analgesics, viscosupplementation as well as joint injection. (JUMMEC $2001 ; 2: 86-91$ )
\end{abstract}

KEY WORDS: Osteoarthritis, management

\section{Introduction}

Osteoarthritis $(O A)$ is the most common joint disorder in the human population. Osteoarthritis is a disorder of synovial joints characterized by destruction of articular cartilage and overgrowth of marginal and subchondral bone. It is the single most important cause for disability and handicap. Factors such as race, genetics, body build, obesity, gender, occupational use, repetitive use and previous injury have all shown to influence this condition.

The pathophysiology of OA can be described as joint failure. Biomechanical stress causes a feedback on the joint cartilage and subchondral bone. This may lead to biochemical changes in the tissues. There is an attempt at joint repair when an injury occurs which may be an anti inflammatory response with cellular infiltrates as well as a fibroblastic response. The repair response incorporates the formation of osteophytes.

There may also be the formation of an effusion followed by thickening of the synovium. Further damage may lead to muscle wasting Later on definite changes such as subchondral sclerosis, osteophytic proliferation and cartilage loss may occur as reflected in classical Xray appearances. These include osteophytes, joint space narrowing, subchondral bony sclerosis, subchondral cysts and mal alignment.

The commonest affected weight bearing joint is that of the knee, in one third of people over age 65 years $(1,2)$; therefore this article will focus mainly on knee OA. Women are twice as likely to suffer from knee OA as men. The COPCORD Study showed that $9.3 \%$ of adult Malaysians complained of knee pain increasing to $23 \%$ in those over 55 years and $39 \%$ in those over 65 years $(3,4)$. The presence of knee OA is more marked than that of hip OA in Asian populations (5).

The main presenting symptom in $O A$ is pain. Other classical symptoms such as "gelling" after inactivity, loss of movement, feelings of instability and functional limitations may occur. Potential sources of pain include osteophyte growth with stretching of the perio steum, raised intraosseous pressure, microfractures, ligament damage, capsular damage, menisceal injury and synovitis due to inflammation. Additional central component factors to pain such as anxiety, depression and co morbidity may play a role in some patients. (6)

The diagnosis of OA is clinical and radiological. Clinical signs include tenderness around the joint margins, crepitus on movement, Heberden's or Bouchard's nodes, effusions, restricted painful movement, quadriceps muscle wasting, deformity or instability of the joints. Weight bearing films of the knee may be required. However Xray changes have a poor correlation with symptoms.

The American College of Rheumatology diagnostic criteria for $O A$ knee include: frequent knee pain for most days over the past month, and one of the following: over 50 years of age, less than $\mathbf{3 0}$ minutes early morning stiffness or crepitus on active movement with osteophytes. (7)

Correspondence:
Emily Goh
Department of Medicine. Faculty of Medicine
University of Malaya, 50603 Kuala Lumpur, Malaysia
Fax: 03 - 795567740
Tel: 03.78502897

Emily Goh

University of Malaya, 50603 Kuala Lumpur, Malaysia

Tel: 03. 78502897 


\section{Management}

The management of OA can be divided into non drug and drug treatment. Non drug management includes measures in primary prevention, physiotherapy, occupational therapy and patient education. Drug management includes the use of various treatments for relief of pain, and finally surgery.

\section{Primary Prevention}

The risk factors in the development of OA include age, heredity, obesity, female preponderance, postmenopausal state and hyper mobility of the joints. Mechanical factors also influence $O A$ such as fractures, menisceal tears and ligament damage as well as abnormalities in joint shape. Certain occupations may also increase the risk oiQOA eg knee OA in manual workers. Primary prevention measures such as weight reduction, prevention of obesity and joint protection techniques (eg avoidance of trauma to joints during sporting activities) are recommended. Those at risk individuals should be identified and advised appropriately.

\section{Non Pharmacologic management}

\section{Exercise and physiotherapy}

A study carried out in Bristol (8) of patients with OA knee noted that quadriceps muscle weakness is the single greatest predictor of loss of lower limb function. The radiographic severity of the joint had no influence in the level of function $(9,10)$

Aerobic deconditioning can also decrease joint stability and function, such as walking and climbing stairs.

Resistance training can improve muscle strength as well reflex inhibition, propioreception and disability $(11,12)$. Resistance training can also retard the progress of joint damage by decreasing the impulse loading on the lower limb. $(13,14)$

Aerobic training can improve joint range of motion, cardiovascular fitness and also co morbidities such as diabetes, hypertension and obesity. $(15,16)$ Psychological variables such as depression, mood disturbances ,emotional health and self efficacy can also be improved. (17-20)

A review of 3 recent trials on aerobic exercise show a modest but significant improvement in disability and also pain control in patients with OA $(2 \mid-23)$. The results were most efficacious in a supervised setting but even home based exercises were helpful to prevent further decline.

The majority of trials ( 1 | out |3) on strengthening exercises also showed a modest but significant improvement in joint disability and pain. (23) Isotonic or closed chain exercises (exercising multiple muscle groups synergistically) such as stepping, squatting are more beneficial (24). Home based quadriceps exercises (25) are also recommended. There is no evidence to suggest that walking or jogging increases the risk of OA (26). Therefore, all patients with OA should be encouraged to do aerobic exercises as well as quadriceps strengthening exercises.

Guidelines for exercises are available in material published by the Arthritis Foundation. (www.arthritis.org), including the PACE I \& PACE II videos and Pathways to Better Living with Arthritis. The Arthritis Foundation of Malaysia also has pamphlets on "Arthritis Needs Exercise" and they run a programme with supervised exercises for patients (www.afkl.org.myl).

Comfortable shoes with shock absorbing insoles can also reduce pain in knee $O A$. Heel wedges can also be used to correct the abnormal biomechanics of the knee (27). Knee braces are also sometimes used to correct deformities. A recent study (28) found reduction in pain when a knee brace was being used. Medial patellar taping may be used in patello-femoral OA (29) Proper use of a walking stick in the contralateral hand can reduce forces of the OA joint by up to $50 \%$ (30) and reduce pain while walking.

Thermal modalities and transcutaneous electrical nerve stimulation can sometimes be used to reduce pain.

\section{Occupational Therapy}

Adaptive equipment, as well as advice on lifestyle and energy saving techniques may be useful in patients with chronic pain. Splinting of affected joints, footwear modification and stress management and relaxation techniques may also be useful in some patients.

\section{Patient education}

Patient education may be an effective way of reducing pain. A study has shown that social support through routine telephone call can reduce pain to the same magnitude as that of an NSAID (3I). In Malaysia patient advice and education leaflets can be obtained from the Arthritis Foundation of Malaysia.

\section{Drugs}

\section{Analgesics}

\section{Non opiod}

Paracetamol should be used as a first line of analgesia. This may relieve pain in up to $20 \%$ of patients but has little effect on physical function (32). A recent study by the Women's Health and Aging study show that this simple analgesia is often underused (33). 


\section{Opiod}

Tramadol (34) is a central acting opiod antagonist which can be used in adjunctive therapy. It can be used in those patients which NSAIDS are contraindicated. Side effects include nausea and vomiting, urinary retention, confusion, drowsiness. It has a very minimal risk of respiratory or central nervous system depression.

\section{Non steroidal anti inflammatory drugs (NSAIDS)}

These drugs may provide symptomatic relief but does not arrest progression. The side effects are well known in this class of drugs including: gastrointestinal (GI) intolerance, $\mathrm{Gl}$ ulceration, perforation and bleeding, platelet aggregation blockade, renal impairment and interstitial nephritis, hypersensitivity reactions and others. There may be considerable variation in response of an individual to different classes of NSAIDS. If a prescribed class is not helpful, a different class should be tried. The analgesic effect should be achieved in one week and the anti -inflammatory effect in three weeks of regular dosing. (35)

It is reasonable to prescribe an NSAIDS on an "as needed" basis leading to the lowest possible effective dosage. If this fails then a regular NSAID for a limited time period (eg 3 weeks) should be prescribed. Precaution should be taken in the elderly and those with renal or hepatic impairment.

The concomitant use of antacids with NSAIDs do not prevent NSAID induced ulcers and may even mask their presence. Prophylaxis with famotidine ( $40 \mathrm{mg}$ daily) can significantly decrease NSAID induced gastric and duodenal ulcers (36) Use of other conventional H2 blockers will reduce duodenal but not gastric induced ulcers (37). Misoprostol with the concomitant use of the proton pump inhibitor (PPI) omeprazole (38) has the best result for ulcer prophylaxis.

Of those who discontinued NSAIDs, $95 \%$ of ulcers can be healed with standard dose ranitidine (39) More recent evidence show that PPI's are superior in ulcer healing (38) (cpg 37) In high risk patients co treatment with PPI's may be recommended.

\section{COX- 2 selective inhibitors}

A major development are the COX2 selective inhibitors. Currently only oral forms of the drug are available but parenteral preparations are being developed. They are no more effective than conventional NSAIDS but have considerably less GI toxicity. The use of these agents leads to a reduction in $\mathrm{Gl}$ perforations, ulcers and bleeds by up to $54 \%(40)$ when compared with conventional NSAIDS. There is no firm evidence that COX -2 NSAIDS will provide additional GI protection if prescribed along with aspirin. The long-term effects of these drugs are still unclear and side effects in the kidney and vasculature sill occur. There is no doubt that these drugs are a safer alternative to conventional NSAIDS especially for those patients at risk of GI toxicity.

Care must be taken in the over prescription of these drugs as well as NSAIDS when simple analgesia or a non-pharmacologic therapy may be equally effective.

\section{Glucosamine}

Glucosamine and chondroitin sulphate are naturally occurring amino monosaccharides in articular cartilage called glycosaminoglycans. Oral supplementation in two recent meta- analysis reported positive outcomes in global pain and functional index (4I). However not all the studies reported positive results and some questions about the design and conduct of these studies remain. A recent study in the published in the Lancet described the long-term effects of glucosamine sulphate on OA progression in a randomized double blind placebo controlled study. Reginster et al (42) compared 212 patients with knee $O A$ in a 3 year follow up with $1500 \mathrm{mg}$ oral glucosamine sulphate daily versus placebo. They found a mean loss of joint space narrowing of $3.1 \mathrm{~mm}$ in the placebo group after 3 years but no significant decrease in mean joint space in the glucosamine treated group. Symptoms of the placebo group also worsened slightly compared to a $20-25 \%$ improvement in symptoms in those who completed the course of glucosamine.

This study suggested that oral glucosamine sulphate may have a long term combined structural and symptom modifying effect in OA. Glucosamine has few side effects and may be a useful addition to the treatment of $\mathrm{OA}$ in some patients.

Further information in the Malaysian context is available in the "Clinical practice Guidelines on the Management of Osteoarthritis", produced by the Malaysian Ministry of Health, Malaysian Society of Rheumatology and Academy of Medicine of Malaysia.

\section{Topical creams/gels}

These have been shown to be more effective than placebo (43) and are in general well tolerated with no increase of side effects.

\section{Intra-articular steroids}

The use of oral steroids has no place in the management of osteoarthritis. The use of intra- articular (IA) steroids have been shown to be more effective than placebo for pain relief (44). IA steroids can be used after aspiration of a joint effusion in OA especially when there is an acute excerbation of joint pain. Sterile tech- 
nique is important and the joint fluid should be sent for microscopy and culture if infection is suspected. General advice to rest the joint for 24 to 48 hours after injection should be given. The minimal time interval between injections should be 3 months.

\section{Inta articular-hyaluronan}

Hyaluronan is a normal component of synovial fluid. It has a role in joint homeostasis as well as joint lubrication, buffers load transmission and provides anti nociceptive and anti inflammatory properties to synovial fluid. It is a glycosaminoglycan (GAG) of molecular weight 4-5 million which is synthesized and released into synovial fluid by specialized synoviocytes. In osteoarthritis, the molecular weight and concentration of hyaluronan are diminished. It has been proposed that removal and replacement of the synovial fluid in an OA joint to restore the molecular weight of hyaluronan will improve joint function. The rational for this is to restore the elastic and viscous properties of synovial fluid as well as normalization of hyaluronan synthesis by synoviocytes. It is also thought that hyaluronan may act by binding onto inflammatory mediators and neuropeptides associated with pain production ${ }^{45}$ This approach is called viscosupplementation.

Some studies $(46,47)$ have shown that the use of intraarticular (IA) hyaluronan for symptomatic knee OA has a modest benefit over placebo or IA steroids for pain relief. However the effect size was not large. Some trials have also shown an effectiveness comparable to continuous oral NSAIDS $(46,48)$ Listrat et al $(49)$ used arthroscopy to score joint cartilage damage at baseline and I year after the use of IA hyaluronan and showed a significant beneficial change on cartilage and reduction of synovial inflammation.

There are a number of different hyaluronan preparations available for knee injection in Malaysia, ie Low molecular weight preparations eg Hyalgan and high molecular weight preparations eg Synvisc. A recent trial comparing these preparations $(50)$ showed better primary outcome results such as weight bearing pain, range of knee movements at one year with the higher molecular weight preparations.

An indication for IA hyaluronan for the knee may be $\mathrm{OA}$ when exercise, non-pharmacologic measures and simple analgesia have not been effective. Viscosupplementation is more effective in early radiographic grades of OA (5I) but also used in more advanced grades.

An important factor is that viscosupplementation is a relatively safe intervention with only very mild and transient local reactions following injection (2-3\% injections). $(52,53)$ Very infrequently more severe local joint reactions occur which may mimic sepsis of the joint.
Fluid should be sent for microscopy and culture if in doubt Proper technique is important when giving the IA injection, if possible the joint should be aspirated beforehand to ensure more efficacy and proper needle placement. About I,000 patients have had IA hyaluronan therapy for a duration of 6 years with no apparent long term effects which gives a good long term safety profile (45).

In summary hyaluronan may have a potential long-term disease modifying effect on $\mathrm{OA}$ as well as a modest effect on pain.

\section{Surgical Procedures}

Those patients with refractory pain despite medical therapy and or progressive limitation in activities of daily living should be referred to an orthopaedic surgeon.

Surgical options include arthroscopic debridement, lavage or abrasion, ligamentous reconstruction, osteotomy, unicompartmental arthroplasty, arthrodesis and total joint replacement. Tidal lavage is effective for some patients with knee OA. One study by Ravaud et al (54) showed benefit in pain relief for 24 weeks. This effect was increased if an IA injection of long acting steroid was given at the same time.

Total joint replacement is of highly beneficial (55) with significant and persistent pain relief, increase in physical function and satisfactory results up to 7 years. The extent of improvement depends on preoperative physical function (56) and the optimum timing for surgery is also important. Total joint arthroplasty is by far the best option in the older age group (above 60). Parameters useful in selecting the best surgical option include: survivorship associated with a given procedure, complications of a procedure and effect of a surgery on subsequent total joint arthroplasty. The patient's age, joints affected, timing of the surgery and expertise available should also be taken into consideration.

\section{Alternative approaches}

\section{Acupuncture}

Acupuncture may be useful in some cases of knee OA. Berman et al (57) gave biweekly acupuncture for patients with knee OA for 8 weeks and found a reduction in pain and disabilty that lasted for 4 weeks after the end of treatment.

\section{Conclusion}

$\mathrm{OA}$ is the commonest joint disorder in the human population. There are now effective therapies to relieve pain and improve function in patients with $O A$. Future research need to be focused on true disease modifying drugs to prevent or stop disease progression in OA which can potentially help millions of sufferers. 


\section{References}

I. Felson D, NaimarkA,Anderson J], et al:The prevalence of knee osteoarthritis in the elderly; Framingham Osteoarthritis study. Arthritis Rheum,987,30:912-918

2 Felson DT : Osteoarthritis. Rheum Clin Dis Clin North Am 1990,16:499-512

3. Veerapen K. "Osteoarthritis - Asian perspective" Textbook of Clinical Rheumatology. (Howe, Feng ed), 294 295 Singapore National Arthritis Foundation, 1997.

4. Veerapen K. Clinical profile of knee pain. Proceedings 9th Asia Pacific League of Associations for Rheumatology Congress. APLAR 2000 pg 119

5. Hoaglund F,T Burlington, Vermont, Arthur CMC, Wong WL. Osteoarthritis of the hip and other joints in Southern Chinese in Hong Kong. J Bone and Joint Surg, April 1973,55A (3):545-547

6. Creamer P, Lethbridge-Cejku M, Hochberg MC : Determinants of pain severity in knee osteoarthritis : effect of demographical and psychosocial variables using 3 pain measures.J Rheumatol 1999,26:1785-1792

7. Altman R. Asche E, Bloch D et al.The ACR criteria for the Classification and reporting of $O A$ of the knee. Arthritis Rheum 1986, 29: 1039-49

8. McAlindon TE, Cooper C. Kirwan JR et al,:Determinants of disability in osteoarthritis of the knee.Ann Rheum Dis $1993,52: 258-262$

9. Slemenda C, Brandt KD, Heilman DK, et al.; Quadriceps weakness and osteoarthritis of the knee.Ann Intern med 1997, 127; 97-104

10. Slemenda C, Heilman DK,Brandt KD, et al. : Reduced quadriceps strength relative to body weight. Arthritis Rheum 1998, 41 : 1951-1959

11. Hurley MV, Scott DL : Improvements in quadriceps sensorimotor function and disability of patients with knee osteoarthritis following a clinically practicable exercise regime. $\mathrm{Br}$ J Rheumatol 1998, 37 :1 181-1187

12. Badley EM, Wagstaff S wood PN : Measures of functional ability (disabilty) in arthritis in relation to impairment of range of joint movement. Ann Rheum Dis 1984, 43: 563 569

13. Radin EL,Parker JW,et al : Response of joints to impact loading, III : relationship between trabecular microfractures and cartilage degeneration.J Biomech 1973 , 6; $51-57$

14. Radin EL,Yang KH, Riegger C, et al.: Relationship between lower limb dynamics and knee joint pain. J Orthop Res 1991, 9: 398-405

15. Campbell WW. Crim MC, Dallas GE et al . : Increased protein requirements in the elderly: new data and retrospective assessments. Am Clin Nutr 1994,60 : 167. 175 .

16. Tononi R: Effect of physical training on the insulin resistance of aging.Am J Physiol 1989, 256 E352-E356

17. RejeskiWJ, EttingerWH, Martin K,et al. : treating disability in knee osteoarthritis with exercise therapy : a central role for self efficacy and pain.Arthritis Care Res 1998,1। 94-101
18. Beniamini Y. Rubenstein JJ ,Zaichkowsly LD,et al. :Effects of high intensity strength training on quality- of -life parameters in cardiac rehabilitation patients.Am J Cardiol $1997,80: 84 \mid-846$

19. McAuley E, Shaffer S, Rudolp D :Physical activity, aging and psychological well being. J Aging Physiolog Activity 1995,3:67.96

20. Singh NA, Clements KM, Fiatarone MA : A randomized controlled trial of progressive training in clinically depressed elders.J Gerontol A Biol Sci Med Sci 1997.52 A :M27-M35

21. Minor MA, Hewett JE,Webel RR et al. efficacy of physical conditioning exercise in patients with rheumatoid arthritis and osteoarthritis. Arthritis Rheum 1989.32: 1396-1405

22. Kovar PA, allegrante Jp, MacKenzier et al.:Supervised fitness walking in patients with osteoarthritis of the knee. Ann Intern Med 1992,116: 529-534

23. Ettinger WH,Burns R, Meissier SP, et al. :A randomized trial comparing aerobic exercise and resistance exercise with a health education program in older adults with knee osteoarthritis. JAMA 1997,227:25-31

24. Baker K, McAlindon T: Exercise for knee osteoarthritis. Current Op Rheum 2000, $12 ; 456-463$

25. O'Reilley SC, Muir KR, Doherty M : effectiveness of home exercise on pain and disability from osteoarthritis of the knee : a randomized controlled study. Ann Rheum Dis 1999.58: 15-19

26. Lane NE, Buckwalter JA : Exercise and osteoarthritis. Curr Opin Rheumatol 1999, 11: 413-416

27. Ogata $K$, Yasunanga M, Nomiyama $H$.The effect of wedged insoles on the thrust of osteoarthritic knees. Int Orthopaed 1997, 21:303-312

28. Komistek RD,Dennis DA, Northcut EJ, et al. : An in vivo analysis of the effectiveness of the osteoarthritic knee brace during heel-strike of gait. J Arthroplasty 1999, 14 : 738-742

29. Cushnaghan J. McCarthy C . Dieppe P.Taping the patella medially: a new treatment for osteoarthritis of the knee joint ?Br J Med. 1994, $308: 753-755$

30. Neumann DA. Hip abductor muscle activity as subjects with hip prosthesis walk with different methods of using a cane. Physical therapy 1998,78(5);490-501

31. Mazzuca SA, Brandt KD, Katz BP : Effects of self care education on the health status of inner city patients with osteoarthritis of the knee. Arthritis Rheum 1997.40: 1466-1474

32. Bradley JD, Brandt KD, Katz BP et al :Comparison of anti inflammatory dose of ibuprofen, an analgesic dose of ibuprofen and acetaminophen in the treatment of patients with OA of the knee. NEJM 1991,325:87-91

33. Pahor M. Guranik JM, Wan JY, et al . : Lower body osteoarticular pain and dose of analgesic medications in older disabled women: The Women's Health and Aging Study. Am J Public Health 1999, $89 ; 930-934$

34. Roth SH : Efficacy and safety of tramadol HCL in breakthrough musculoskeletal pain attributed to OA.J Rheumatol 1998.25: 1358-1363 
35. British National formulary, March 2001

36. Taha As, Hudson N, Hawkey C), et al.; Famotidine for the prevention of gastric and duodenal ulcers caused by non steroidal anti- inflammatory drugs. N Eng J Med 1996:334; 1435-9

37. Ehsanullah RS, Page MC.Tildesly G,Wood JR.; Prevention of gastroduodenal damage by non steroidal antiinflammatory drugs :controlled trial of ranitidine. BMJ 1998, 297:1017-21

38. Hawkey CJ. Karrasch JA, Szczepanski L, et al.;Omeprazole compared with misoprostol for ulcers associated with non -steroidal anti -inflammatory drugs. N Eng J Med 1998,338:727-34

39. Lancaster- Smith MJ, jadeberg MR, Jackson DA. Ranitidine in the treatment of non-steroidal anti-inflammatory drug associated gastric and duodenal ulcers. Gut 1991,32: 252-6

40. Bombardier C, Laine L, Reicin A et al : Comparison of upper gastrointestinal toxicity of rofecoxib and naproxen in patients with rheumatoid arthritis. NEJM 2000,343 : 1520-28

41. Towheed TE, Mcmillan R, Wein C, et al. : effect of glucosamine hydrochloride on the treatment of pain in OA of the knee.J Rheumatol 1999,26:2423-2430

42. Reginster JY, Deroisy R, Rovati LC, et al.; Long term effects of glucosamine sulphate on osteoarthritis progression ; a randomized, placebo-controlled clinical trial. Lancet 2000,35(9252) $7: 251-256$

43. Moore RA, Tramer M, Carroll D, et al. ; Quantitative systemic review of topically applied non steroidal antiinflammatory drugs. BMJ 1998,316;333-338

44 Sathapatayavongs B, Jones HE, Bacon PA, Ring EF, Dieppe PA. Intra-articular $\$$ teroids in osteoarthritis. Rheumatol Rehabil.1980,19(4): 212-17

45. Wayne Marshall K. Intra-articular hyaluronan therapy. Curr Op Rheum 2000,12:468-474

46. Altman RD, Moskowitz R : Intra-articular sodium hyaluronate (Hyalgan) in the treatment of patients with osteoarthritis of the knee: a randomised clinical trial. J Rheumatol 1998,25:2203-2212

47. Huskisson EC, Donelly S. Hyaluronic acid in the treatment of osteoarthritis of the knee. Rheumatology 1999,38 ; $602-7$
48. Adams ME, atkinson $M H$, Lussier AJ,et al. : The role of viscosupplementation with hylan G-F 20 (Synmvisc) in the treatment of osteoarthritis of the knee : a Canadian multicentre trial comparing hylan G-F 20 alone, hylan GF 20 with NSAIDs and NSAIDS alone. Osteoarthritis Cartilage 1995,3:213-225

49 Listrat V,Ayral X,Patarnello F,etal.:Arthroscopic evaluation of potential s. tructure modifying activity of hyaluronan (Hyalgan) in osteoarthritis of the knee. Osteoarthritis Cart 1997.5: 153-160

50. Wobig M, Bach G, Beks P et al.The role of elastoviscosity in the efficacy of viscosupplementation for OA of the knee : A comparison between hylan G-F 20 and a lowermolecular- weight hyaluronan. Clin Ther 1999,21:15491562

51. Balazs EA, Briller SO, Denlinger JL. : Na- hyaluronate molecular size variations in equine and human arthritic synovial fluids and the effects on phagocytic cells. In Seminars in arthritis and reumatism,volll. Edited by Talbott Jh. New York: Grune \& Stratton; 1981:|41-143

52. Lussier A, CividoAA, Mc Farlane Ca,et al. : Viscosupplementation with hylan for the treatment of osteoarthritis : findings from clinical practice in Canada. J Rheumatol 1996,23: 1579-1585

53. Wobig M, Beks P, Dickhut A,et al. Open-label multicentre trial of the safety and efficacy of viscosupplementation with hylan G-F 20 (synvisc) in primary osteoarthritis of the knee. J Clin Rheumatol 1999,5 (suppl): S24-S3I

54. Ravaud P, Mouliner L, Giraudeau B,et al. : Effects of joint lavage and steroid injection in patients with OA of the knee : results of a multicentre, randomized, controlled trial.Arthritis Rheum 1999,42: 475-482

55. Hawker G,Wreight J, Coyte $P$ et al.: Health- related quality of life after knee replacement.J Bone Joint Surg (US) 1998,80: 163-173

56. Fortin PR, Clarke AE, Joseph L, et al.: Outcomes of total hip and knee replacement.Arthritis Rheum 1999,42 : 17221728.

57. Berman BM, Singh B, Lao L, et al:: A randomized trail of acupuncture as an adjunctive therapy in OA of the knee. Rheumatology 1999,26:2294-2297 